\title{
The therapeutic effects of traditional Chinese medicine Fusu agent in LPS-induced acute lung injury model rats
}

This article was published in the following Dove Press journal: Drug Design, Development and Therapy

\author{
Peiyang Gao',* \\ Ziyi Zhao ${ }^{2, *}$ \\ Chuantao Zhang' \\ Chunxia Wang' \\ Kunlan Long' \\ Liuxue Guo' \\ Baixue $\mathrm{Li}^{3}$ \\ 'Intensive Care Unit, The Teaching \\ Hospital of Chengdu University \\ of Traditional Chinese Medicine, \\ Chengdu, China; ${ }^{2}$ Central Laboratory, \\ The Teaching Hospital of Chengdu \\ University of Traditional Chinese \\ Medicine, Chengdu, China; ${ }^{3}$ College of \\ Basic Medicine, Chengdu University \\ of Traditional Chinese Medicine, \\ Chengdu, China \\ *These authors contributed equally \\ to this work
}

\begin{abstract}
Purpose: Acute lung injury (ALI) is a common and fatal oxidative stress in the lung, mainly induced by endothelial injury and capillary leakage. In our previous study, "Fusu agent", a traditional Chinese medicine, was found to exert preventive effect on endothelial damage in lipopolysaccharide (LPS)-induced ALI model rats partially via inhibiting heparanase1 (HPA1) activation and inhibiting the inflammatory factors. However, it is still unknown whether Fusu agent exerts its therapeutic effect in LPS-induced ALI model rats and its potential mechanism.
\end{abstract}

Materials and methods: Rats were injected with LPS ( $3 \mathrm{mg} / \mathrm{kg}$, intraperitoneally) to induced ALI, and the prepared Fusu agent was given (2, 4 or $6 \mathrm{~g} / \mathrm{kg}) 2$ hours after LPS challenge. Twenty-four or 48 hours after Fusu agent administration, the biochemical changes in the plasma and lung tissues and the morphological/histological changes in the lung associated with inflammation and injury were evaluated. Human umbilical vein endothelial cells (HUVECs) were employed to confirm the therapeutic effects of Fusu agent and investigate its mechanisms, that is, affecting ROS accumulation, mitochondrial transmembrane potential (MTP) maintenance and decreasing the expression levels of HPA1.

Results: Administration of Fusu agent obviously improved the lung injury and recovered vascular endothelium loss and injury. CD31 signal, which is a specific marker for endothelial vascular lesions, was decreased after Fusu agent treatment in LPS-induced ALI model rats, indicating its therapeutic effect against endothelial surface layer injury. Meanwhile, Fusu agent also decreased HPA1 expression and inflammatory responses. In vitro, Fusu agent-medicated serum decreased injury and cell death induced by LPS in HUVECs by stabilizing MTP and decreasing the leakage of lactate dehydrogenase. Consistently, Fusu agent-medicated serum downregulated HPA1 induced by LPS stimulation.

Conclusion: These findings suggest that Fusu agent exerts its therapeutic effect in both LPSinduced ALI model rats and HUVECs potentially via suppressing HPA1 expression, and thus exerts prosurvival effect via maintaining MTP and attenuating cell injury.

Keywords: ALI, heparanase, mitochondrial transmembrane potential, HUVECs, acute lung injury, ROS accumulation

\section{Introduction}

Acute lung injury (ALI), which occurs in nearly 2,000,000 patients worldwide every year, is mainly associated with the lung and leads to a low oxygen level in the blood. ${ }^{1,2}$ ALI is characterized histologically by diffuse inflammatory alveolar infiltration, hemorrhage, pulmonary edema, and hyaline membrane formation. ${ }^{3,4}$ In the past two decades, several therapeutic strategies have been developed and remarkable progress has been made; however, the mortality rate remains high, where over a third of sufferers die, and 
some of the survivors present complications such as brain damage because of prolonged low level of oxygen ${ }^{5,6}$ Increased permeability of the alveolar capillary membrane plays a central role in the pathophysiological mechanism of ALI. ${ }^{4}$ Intensive inflammatory responses are the main cause of ALI. Sepsisrelated ALI is especially a common, costly disease with a high mortality rate and no targeted therapies; 5,7 by causing indirect (extrapulmonary or secondary) lung injury, it damages the vascular endothelium. ${ }^{1,5}$ Lipopolysaccharide (LPS) stimulation has been widely accepted as an efficient strategy for establishing clinically relevant animal model of ALI by causing inflammation. ${ }^{89}$ Thus, LPS-induced ALI model animals are widely used for the preclinical investigation of ALI.

Glycocalyx, which lies between the flowing blood and endothelial cells, is a complex gel with a protective effect. In pulmonary endothelial cells, the endothelial glycocalyx is a heparin sulfate (HS)-rich layer of glycosaminoglycan and associated proteoglycans. ${ }^{10}$ Pulmonary heparanase1 (HPA1) specifically degrades pulmonary endothelial glycocalyx by its HS-specific digesting activity. Upregulation and activation of HPA1 promotes inflammatory effects by causing endothelial dysfunction, inflammatory lung injury, and upregulating tumor necrosis factor (TNF) or interleukin (IL)-1 $\beta$ in pulmonary endothelial cells. ${ }^{11,12}$ Activation of HPA1 is associated with ALI as it leads to degradation of pulmonary endothelial glycocalyx and causes endothelial dysfunction and inflammatory lung injury. ${ }^{10}$

LPS stimulation causes inflammatory response and cell injury in human umbilical vein endothelial cells (HUVECs) and is well accepted as a strategy for establishing an in vitro model of ALI. ${ }^{13,14}$ In HUVECs, LPS stimulation causes inflammatory cytotoxicity by promoting the inflammation processes and causing reactive oxygen species (ROS) and nitric oxide (NO) accumulation, which are eliminated by pretreatment with scavenger of ROS or NO. ${ }^{15}$ It is reported that, the ROS accumulated by LPS stimulation are potentially the key factors that cause irreversible damage to ALI model rats, disabling the mitochondrial membrane potential. ${ }^{16}$ This is consistent with previous report showing that excessive ROS can cause mitochondrial dysfunction and leads to caspase-3-dependent apoptotic cell death. ${ }^{17,18}$ By considering these results, in this study, HUVECs were employed for investigating the therapeutic effects of Fusu agent on LPS-induced cell injury.

Traditional Chinese medicine (TCM), which has developed over thousands of years, is based on distinct principles and foundation from western medicine and has been widely used. TCM formulae are usually effective alternatives to western medicine for uncertain disorders, including ALI. ${ }^{19,20}$ For treating ALI, TCM follows the therapeutic concepts of integrated and balanced treatment. Fusu agent is a newly developed oral medicine used in clinical practice in China to treat sepsis-associated ALI. Fusu agent, which is made from Aconitum carmichaelii Debx, Carapax Testudinis, Fructus Amomi, Rhizome Zingiberis, Radix Glycyrrhizae Preparata and Herba Ephedrae, has been clinically used in China for curing ALI. In our previous study, it was revealed that pretreatment with Fusu agent inhibits the inflammatory factors, attenuates lung capillary leak and improves the clinical effect in LPS-induced ALI model rats. ${ }^{21,22}$ The following mechanism investigation reveals that, by downregulating the expression level of HPA1, Fusu agent inhibits the inflammatory cell influx and neutrophil adhesion in ALI. However, how Fusu agent exerts its therapeutic effect in LPS-induced model rats is still unknown. In this study, we further investigated the therapeutic effects of Fusu agent on LPS-induced ALI model rats and its potential mechanism.

\section{Materials and methods Preparation of Fusu agent}

Fusu agent extract from six species of medical herbs, $A$. carmichaelii Debx, Carapax Testudinis, Fructus Amomi, Rhizome Zingiberis, Radix Glycyrrhizae Preparata and Herba Ephedrae, was prepared as described previously. ${ }^{23}$ All drug materials were purchased and identified according to the rigid specifications set by the Chinese Pharmacopeia (2015 Edition). Briefly, the above materials were concerted in a ratio of 6:3:4:3:2:3. First, $A$. carmichaelii Debx and Carapax Testudinis were soaked for 30 minutes in water and decocted for 30 minutes. Then, the other four species of medical herbs (Fructus Amomi, Rhizome Zingiberis, Radix Glycyrrhizae Preparata and Herba Ephedrae) were added and decocted three times with boiling distilled water for 1 hour. The decoction was filtered, collected and concentrated to an evenly thick paste. The thick paste was dried in an oven for 48 hours and pounded into powder. In this experiment, the dose of Fusu agent for animal was the dose of the crude drug.

Previously, the chemical constituents of Fusu agent extract had been identified. ${ }^{23}$ For analyzing the quality of the newly prepared Fusu agent, the fingerprint of the Fusu agent was determined by HPLC (Agilent Technologies 1200 Series). As expected, 21 characteristic peaks were found. Three characteristic peaks, including liquiritin, glycyrrhizic acid and 6-gingerol, were identified by comparing with the reference standard, respectively. The peak for liquiritin was considered as the quality control, which indicated good 
separation without impurity interference (data not shown), and the acceptable consistency of Fusu agent.

\section{Animals}

A total of 72 male Wistar rats, 8-10 weeks old and weighing 220-250 g, were purchased from Chengdu Dashuo Experimental Animal Cooperation. The rats were maintained on a standard diet, and water was made freely available. According to the basis of experimental methodology of pharmacology, ${ }^{24}$ the rats were randomly divided into five groups: 1) untreated animals (control group, $\mathrm{NC}$; $\mathrm{n}=8$ ); 2) LPS-induced model group (rats treated with intraperitoneal [i.p.] injection of $3 \mathrm{mg} / \mathrm{kg}$ of LPS [cat. no: L3129; SigmaAldrich, St Louis, MO, USA], M; n=16); 3) low-dose Fusu agent and ALI group (Fusu agent was administered with stomach perfusion at a daily dose of $2.0 \mathrm{~g} / \mathrm{kg}$ for 2 days and 2 hours after i.p. injection of $3 \mathrm{mg} / \mathrm{kg}$ of LPS, LD; $\mathrm{n}=16$ ); 4) middle-dose Fusu agent and ALI group (Fusu agent was administered with stomach perfusion at a daily dose of $4.0 \mathrm{~g} / \mathrm{kg}$ for 2 days and 2 hours after i.p. injection of $3 \mathrm{mg} / \mathrm{kg}$ of LPS, MD; n=16) and 5) high-dose Fusu agent and ALI group (Fusu agent was administered with stomach perfusion at a daily dose of $6.0 \mathrm{~g} / \mathrm{kg}$ for 2 days and 2 hours after i.p. injection of $3 \mathrm{mg} / \mathrm{kg}$ of LPS, HD; $\mathrm{n}=16$ ). After the injection of LPS, the body mass and breathing of all rats were monitored every 6 hours. When both clinical signs were observed, rats were immediately euthanized. After 48 hours, all rats were euthanized and the lung tissue was separately stored at $-80^{\circ} \mathrm{C}$ or fixed in formalin over 24 hours for sectioning. The blood was obtained from the main artery in all groups and plasma was isolated by centrifugation $(12,000 \times g, 15$ minutes $)$ and stored at $-80^{\circ} \mathrm{C}$.

\section{Cell culture}

HUVECs were obtained from the American Type Culture Collection (ATCC, Manassas, VA, USA) and cultured in DMEM supplemented with 10\% FBS (Thermo Fisher Scientific, Waltham, MA, USA), $100 \mathrm{U} / \mathrm{mL}$ penicillin and $100 \mathrm{~g} / \mathrm{mL}$ streptomycin in a humidified atmosphere of $5 \% \mathrm{CO}_{2}$ and $95 \%$ air at $37^{\circ} \mathrm{C}$. For LPS exposure, $1 \mu \mathrm{g} / \mathrm{mL}$ LPS was added for an extra 6-hour co-incubation and the medium was refreshed. The mock group was treated with the medium without LPS for 6-hour co-incubation.

\section{Survival curves}

To assess the effect of Fusu agent on survival, observations of survival were performed at 24 and 48 hours, respectively. If the rat presents body mass loss over $30 \%$ and labored breathing, for minimizing animal suffering, the rat was considered to have reached the experiment end point and was euthanized immediately according to the approved protocol.

\section{H\&E staining}

After all the rats were sacrificed, lung tissue samples were fixed in $10 \%$ buffered formalin for 24 hours and then dehydrated by washing in ascending grades of ethanol. Samples were then sectioned and embedded in paraffin wax for H\&E staining. The stained sections were observed for morphological changes. Images were taken under $200 x$ magnification at five random fields of view under an X71 (U-RFL-T) fluorescence microscope (Olympus, Melville, NY, USA). The lung tissues were collected to measure the lung injury score by histopathologic evaluation.

\section{Immunohistochemistry}

The expression of CD31 in the lung tissues was detected by immunohistochemistry. The sectioned lung tissues were deparaffinized using xylene and dehydrated by graded concentrations of alcohol; they were then placed in a retrieval solution and incubated for 30 minutes in a microwave at high power. Followed by a cooling process, the slides were washed with PBS three times and incubated sequentially with the primary antibody (ab119339; Abcam, Cambridge, UK) at a dilution of 1:50 and biotin-labeled secondary antibody (ab47844; Abcam) at a dilution of 1:1,000. Finally, the slides were stained with 3,3'-diaminobenzidine, counterstained with hematoxylin, dehydrated, cleared in xylene and fixed. Images were taken under $200 \times$ magnification at five different microscopic fields (X71 [U-RFL-T] fluorescence microscope; Olympus).

\section{Drug serum and control serum preparation}

Ten male Wistar rats, specific pathogen-flee grade, weighing 220-250 g, were divided equally into control and medicated groups. The medicated group received Fusu agent at a dose of $6.0 \mathrm{~g} / \mathrm{kg}$, twice daily for 7 days. After the last treatment, serum was collected and inactivated at $56^{\circ} \mathrm{C}$ for 30 minutes and stored at $-80^{\circ} \mathrm{C}$ for long-term use.

\section{Apoptotic cell death detection}

Cells were harvested, washed and resuspended with PBS. Apoptotic cells were determined with a fluorescein isothiocyanate Annexin V Apoptosis Detection Kit (BD Biosciences, San Jose, CA, USA) according to the manufacturer's 
protocol. Briefly, the prepared cells were incubated for 15 minutes at room temperature in the dark in $100 \mu \mathrm{L}$ of $1 \times$ binding buffer supplemented with $5 \mu \mathrm{L}$ of Annexin V-fluorescein isothiocyanate and $5 \mu \mathrm{L}$ of propidium iodide (PI). Afterward, the cells were pelleted and the supernatant was replaced with $1 \times$ binding buffer and then analyzed by three laser Navios flow cytometers (Beckman Coulter, Brea, CA, USA).

\section{Lactate dehydrogenase (LDH) detection}

For detecting the release of $\mathrm{LDH}$, the medium from each culture was saved in a $1.5 \mathrm{~mL}$ microtube, followed by $\mathrm{LDH}$ determination using Sigma Diagnostic Kit (Sigma-Aldrich). Fifty microliters of the sample was incubated with $500 \mu \mathrm{L}$ of the reagent, and LDH was quantified spectrophotometrically by the rate of change in absorbance at $340 \mathrm{~nm}$.

\section{ROS detection}

The cells were harvested, washed, and resuspended and incubated with $100 \mathrm{ng} / \mathrm{mL}$ dihydroethidium for 30 minutes in the dark. ROS were measured by a microplate reader (Synergy 2 Multi-Mode Microplate Reader; BioTek, Winooski, VT, USA) at excitation and emission wavelengths of 485 and $530 \mathrm{~nm}$, respectively.

For siRNA transfection, siRNA was employed for knocking down the expression of HPA1 in HUVECs. A 21-nucleotide siRNA targeting transcription start site (TSS, -9/+10 bp, 5'-GGGCGGAGGAAAGGAGAAAtt-3') were chosen for HPA1 silencing. ${ }^{19}$ The siRNA was chemically synthesized and transfected with Lipofectamine 2000 (Thermo Fisher Scientific) according to the suggested concentration of manufacturer (RiboBio Co. Ltd, Guangzhou, China).

\section{Statistical analysis}

All data are expressed as the mean \pm SD. One-way ANOVA followed by Bonferroni's multiple comparison test were used for comparisons among experimental groups using GraphPad Prism 5 software (GraphPad Inc., La Jolla, CA, USA). A $P$-value $<0.05$ was considered statistically significant.

\section{Ethics statement}

This study was carried out in strict accordance with the recommendations in the "Guide for the Care and Use of Laboratory Animals" published by the National Institutes of Health. The protocol was approved by the Experimental Animal Ethics Committee of the Affiliated Hospital of Chengdu University of Traditional Chinese Medicine (protocol number: 2017
DL-001). All surgeries were performed under sodium pentobarbital anesthesia (30 mg/kg, intravenous), and all efforts were made to minimize suffering. A strict human end point was set when the animals showed $30 \%$ body mass loss and labored breathing. When the rats showed both the above-mentioned clinical signs, they were considered to have reached the end point of the experiment and were euthanized immediately according to the protocol of the Experimental Animal Ethics Committee of the Affiliated Hospital of Chengdu University of Traditional Chinese Medicine.

\section{Results}

\section{Fusu agent histopathologically attenuated the severity and inflammatory response in LPS-induced ALI model rats}

By tail vein injection of $3 \mathrm{mg} / \mathrm{kg}$ LPS after 2 hours, rats were garaged with Fusu agent for 48 hours at different concentrations and then sacrificed for collecting the serum and lung tissues. As shown in Figure 1A, LPS administration obviously thickened the alveolar walls and congested the capillaries with numerous polymorphonuclear leukocytes, which are the pathological effects of LPS injection. Treatment with Fusu agent obviously attenuated the pathological changes at all three concentrations, and high concentration of Fusu agent gave better results (Figure 1A).

Then the severity of LPS-induced lung injury was determined according to Smith score. In Figure 1B, quantal scoring of histological lung injury in $\mathrm{M}$ group was found to be significantly higher than that of NC group at both 24 and 48 hours ( ${ }^{\triangle} P<0.05$ vs $\mathrm{NC}$ group), indicating that the ALI rat model was established successfully. Administration of model rats with Fusu agent significantly reduced the quantitative scoring of histological lung injury $\left({ }^{*} P<0.05\right.$ vs $M$ group). Moreover, the survival rate of Fusu agentadministered groups was also significantly higher than that of $\mathrm{M}$ group (Figure 1C). Together, these data showed that treatment with Fusu agent presented therapeutic effects in LPS-induced ALI model rats, although the lung injury was not completely healed.

\section{Fusu agent decreased endothelial surface layer (ESL) injury}

By resulting alveolo-capillary barrier leakage, ESL injury is recognized as the initial event. For further investigating whether Fusu agent exerts its therapeutic effects on ALI model rats, CD31 (endothelial cell adhesion molecule-1), which is recognized as a specific marker for endothelial vascular lesions, ${ }^{25}$ was employed for evaluating lung injury 


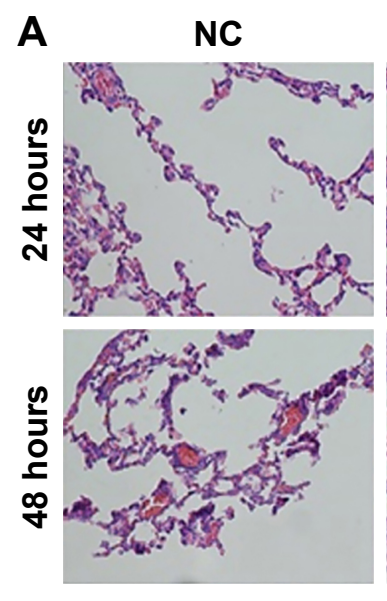

B

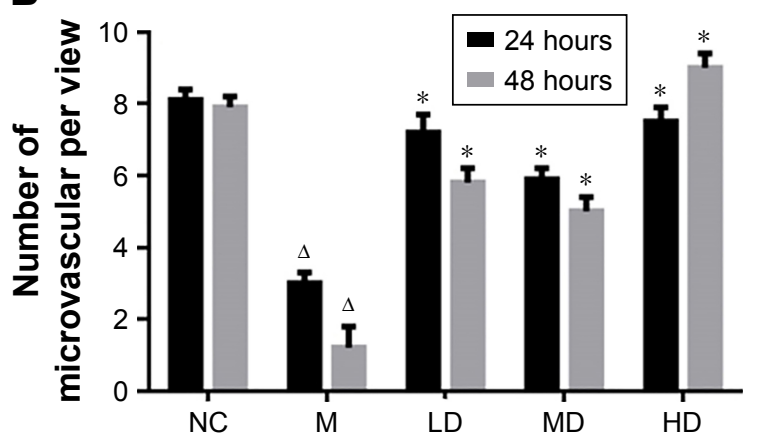

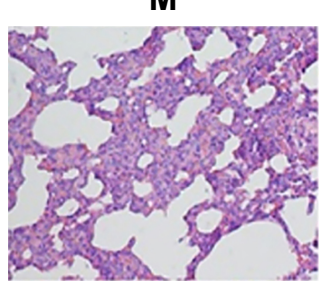
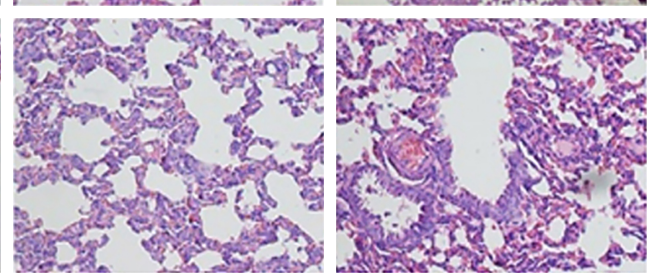

LD

MD
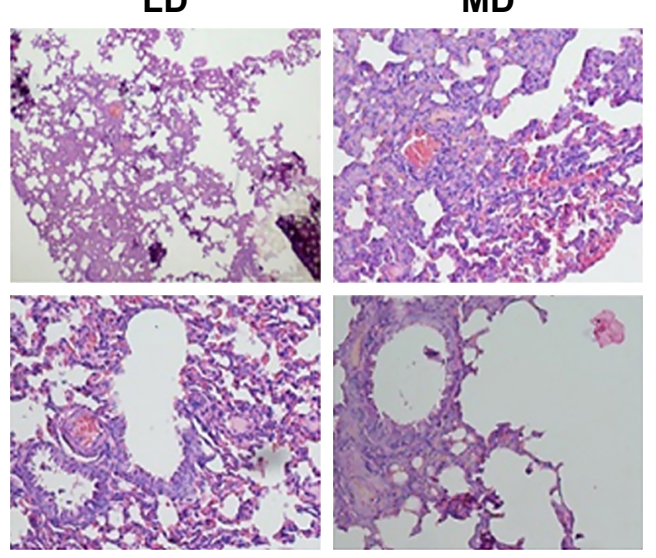

HD
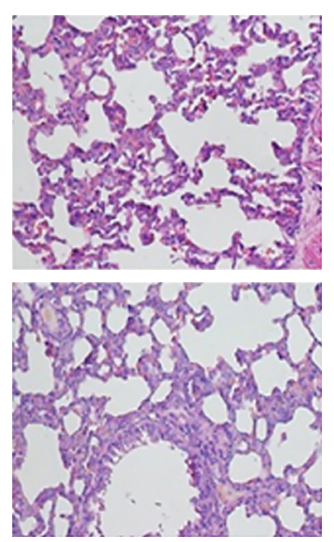

C

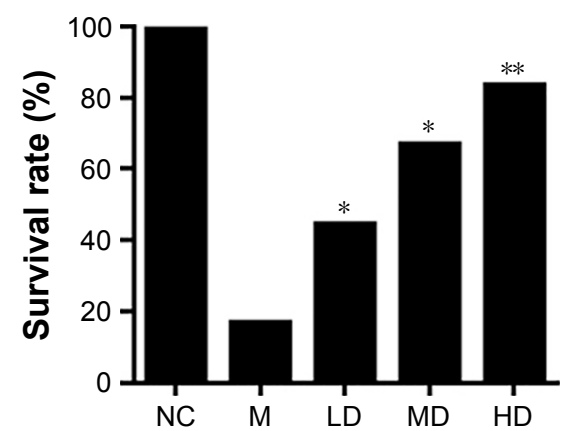

Figure I Fusu agent exerted its therapeutic effects on LPS-induced ALI model rats.

Notes: (A) Histological structures of NC, M, LD, MD and HD groups at 24- and 48-hours time points after LPS stimulation determined by H\&E staining (original magnification, $\times 200$ ). (B) Lung injury score was determined and graded according to Matute-Bello scoring system. $* P<0.05$ vs $M$ group; $\triangle P<0.05$ vs $N C$ group. (C) Survival rate of rats at 48 hours. $* P<0.05$ vs $M$ group at 48 hours, $* * P<0.01$.

Abbreviations: ALI, acute lung injury; LPS, lipopolysaccharide; NC, negative control group; M, model group; LD, low dose; MD, middle dose; HD, high dose.

after Fusu agent administration. In LPS-induced ALI model group, positive signal was clearly observed and pulmonary endothelial cells decreased compared with the NC group. In contrast, Fusu agent administration in LPS-induced model group showed clearer vascular endothelium and vascular integrity (Figure 2), demonstrating that Fusu agent can reduce the loss and injury of vascular endothelium.

\section{Effects of Fusu agent on the expression of HPA, TNF- $\alpha$ and IL- 10 in plasma}

IL-10 is one of the most crucial immunoregulatory cytokines which plays a role in the maintenance of immune homeostasis. ${ }^{26} \mathrm{TNF}-\alpha$ plays an important role in the inflammatory responses. ${ }^{27}$ In our previous reports, it has been shown that both IL-10 and TNF- $\alpha$ are upregulated after
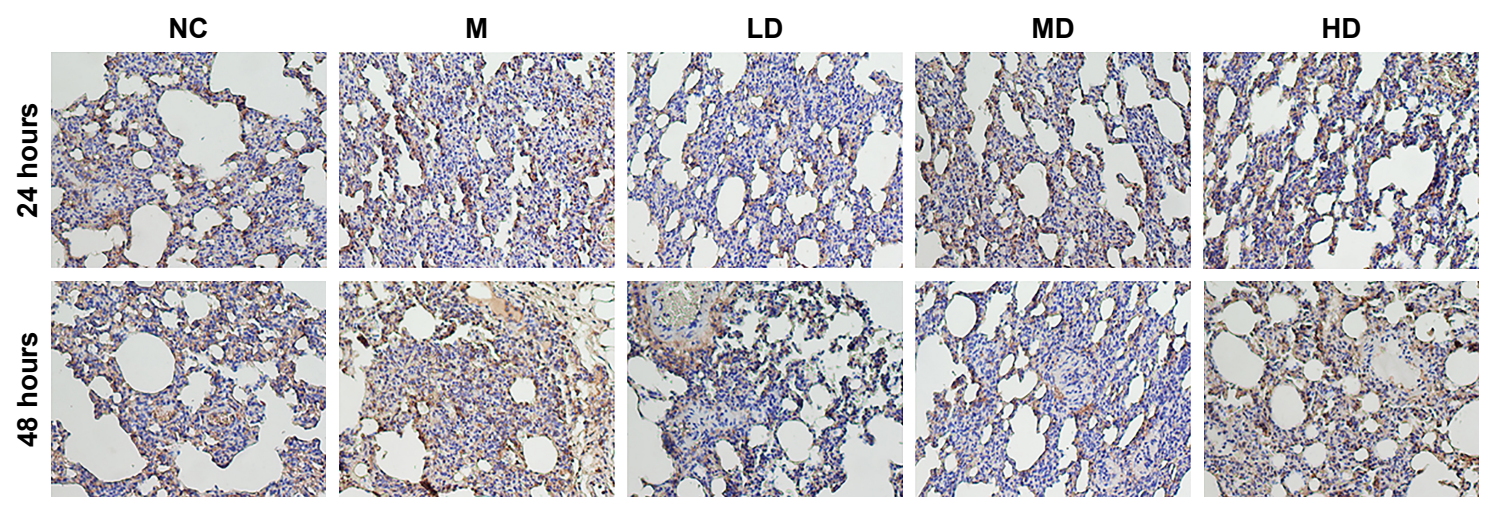

Figure 2 Fusu agent inhibited LPS-induced endothelial vascular injury.

Notes: Immunohistochemical staining for CD3I, which presents the endothelial vascular lesions specifically. Magnification $\times 100$.

Abbreviations: LPS, lipopolysaccharide; NC, negative control group; M, model group; LD, low dose; MD, middle dose; HD, high dose. 
LPS stimulation and regulated inflammatory responses, and Fusu agent administration exerted protective effects by decreasing the levels of IL-10 and TNF- $\alpha$ in plasma. ${ }^{22,23}$ For identifying the effects of Fusu agent on LPS-induced inflammatory responses, the levels of IL-10 and TNF- $\alpha$ in plasma were measured. As shown in Figure $3 \mathrm{~A}$ and B, after LPS stimulation, TNF- $\alpha$ and IL-10 levels increased remarkably as expected. In contrast, Fusu agent could significantly decrease the expression of these inflammatory cytokines, demonstrating that Fusu agent successfully inhibited the inflammatory responses. To demonstrate the effects of Fusu agent on HPA1, we then measured the level of HPA1 in plasma. As expected, LPS stimulation upregulated the level of HPA1 in plasma (Figure 3C). Conversely, Fusu agent administration decreased HPA1 level in LPS-induced ALI model rats, indicating that HPA1 is a potential therapeutic target of Fusu agent.

\section{Fusu agent regulates HPAI, Syndecan-I, Glypican I and Rho A proteins significantly}

In our previous reports, HPA1, Syndecan-I and Glypican1, which are all necessary for ESL mediation, were found to be tightly regulated by pretreatment with Fusu agent. ${ }^{22,23} \mathrm{By}$ considering the involvement of ICAM1 and ROCK1/Rho A in regulating intracellular adhesion and motility, ${ }^{28,29}$ we then measured the effects of Fusu agent on these protein levels in LPS-induced model rats' lung tissue. Western blot was performed at 24 and 48 hours after Fusu agent administration (Figure 4). At 24 hours, LPS stimulation obviously upregulated HPA1, Syndecan-I, Glypican1 and Rho A, and in contrast, Fusu agent administration obviously decreased these protein levels, without detectable disturbance on ICAM1 and
ROCK-I. Notably, at 48 hours, except HPA1, all proteins were undetectably affected by LPS stimulation.

\section{Fusu agent-medicated serum exerted therapeutic effects in LPS-stimulated HUVECS}

For further confirming the effects of Fusu agent on vascular endothelial cells, HUVECs stimulated with LPS were treated with Fusu agent-medicated serum. As previously reported, ${ }^{30,31}$ LPS stimulation induced accumulation of ROS, leakage of LDH, decreased mitochondrial transmembrane potential (MTP) and promoted apoptotic cell death by activating caspase-3 activity (Figure 5A-E) as compared with the NC group. Without disturbing ROS generation, Fusu agent-medicated serum stabilized MTP and decreased the leakage of LDH, and thus inhibited apoptotic cell death (Figure 5A-E).

In the context of ALI, both apoptotic and necrotic cell death, which cause damage to organs, are induced by inflammatory responses. This promoted us to detect whether necrosis is regulated by Fusu agent-medicated serum after LPS stimulation. High-dose Fusu agent-medicated serum was employed for investigating its regulatory roles on apoptosis and necrosis because of the previous demonstrated data showing that high-dose Fusu agent-mediated serum had obvious therapeutic effects (Figures 1 and 2). For determining cell death rate, Annexin V/PI staining was performed; $\mathrm{PI}+/$ Annexin V-cells were considered as necrotic cells, PI-/ Annexin V+ cells as apoptotic cells and PI+/Annexin V+cells were considered as necrotic and apoptotic cells. As shown in Figure 6A and B, LPS stimulation increased both PI+/ Annexin V-cells and PI-/Annexin V+cells, indicating that LPS stimulation caused cell injury via inducing both apoptosis and necrosis as expected. Treatment of high-dose
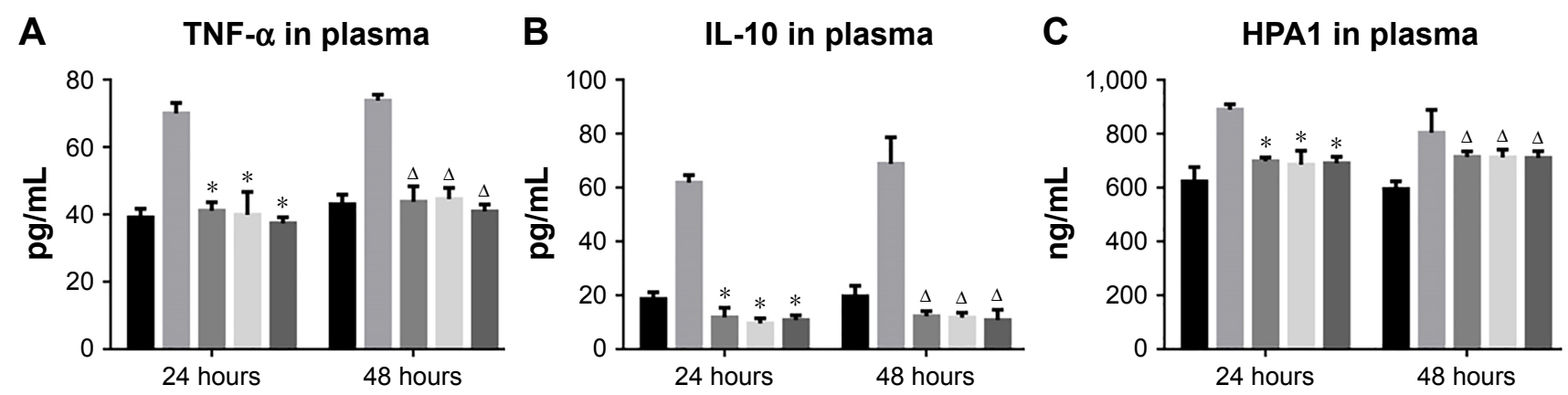

m NC $\quad M \quad$ LD $\quad$ MD $\quad$ HD

Figure 3 Fusu agent downregulated HPAI, TNF- $\alpha$ and IL- 10 in plasma.

Notes: By performing ELISA, the levels of (A) HPAI, (B) TNF- $\alpha$ and (C) IL- 10 were measured. $* P<0.05$ vs $M$ group; ${ }^{\wedge} P<0.05$.

Abbreviations: TNF, tumor necrosis factor; HPAI, heparanase I; IL, interleukin; NC, negative control group; M, model group; LD, low dose; MD, middle dose; HD, high dose. 


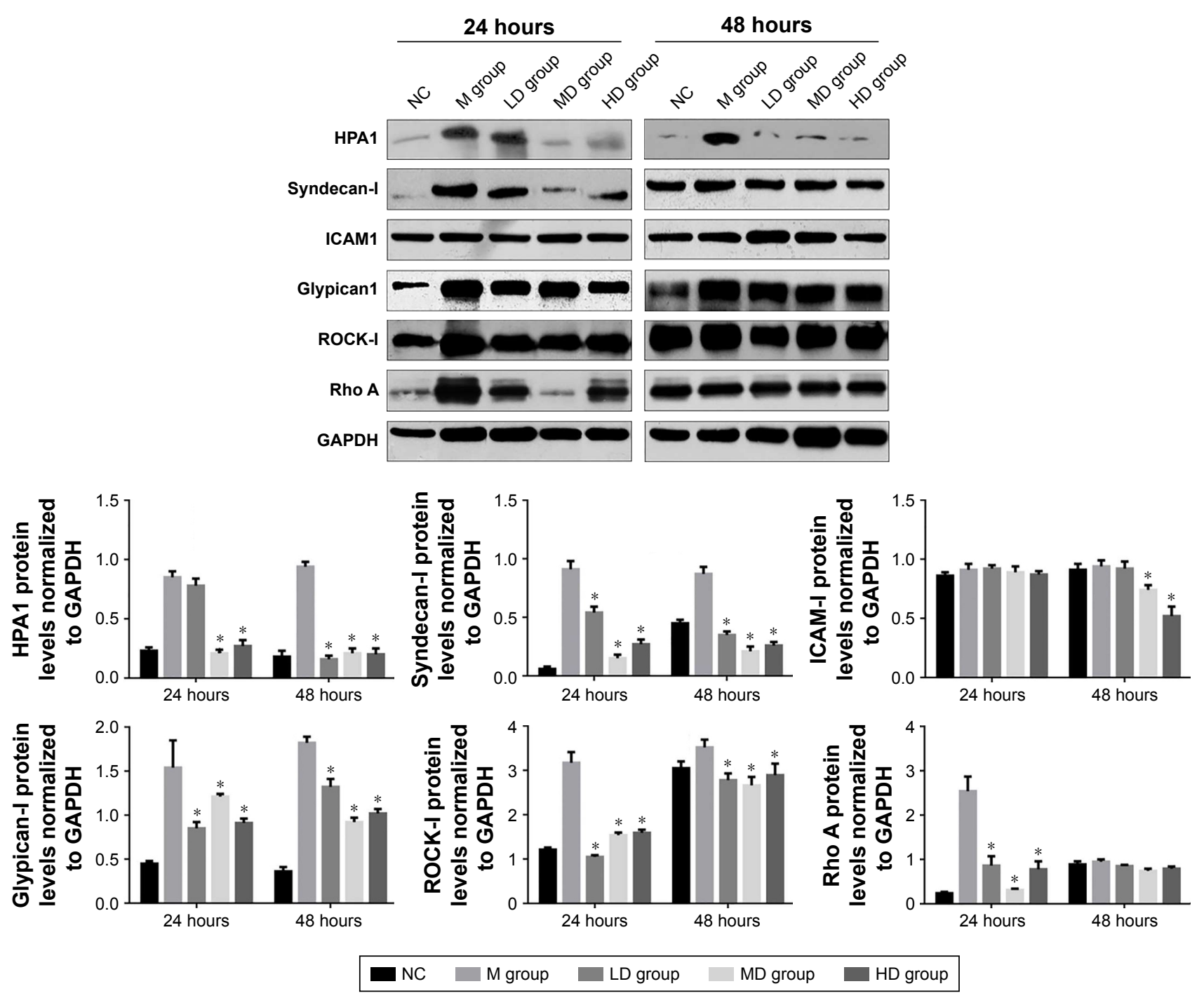

Figure 4 Fusu agent downregulated HPAI, Syndecan-I, ICAMI, Glypican I, ROCK-I and Rho A protein levels in the lung tissues.

Notes: By performing semi-quantitative Western blot, the HPAI, Syndecan-I, ICAMI, GlypicanI, ROCK-I and Rho A protein levels in the lung tissues were measured and normalized to GAPDH protein level. $* P<0.05$ vs $M$ group.

Abbreviations: HPAI, heparanase I; NC, negative control group; M, model group; LD, low dose; MD, middle dose; HD, high dose.

Fusu agent-medicated serum further attenuated injury in either z-Val-Ala-Asp(Ome)-fluoromethylketone or necrosulfonamide-pretreated cells, indicating that it protected the cells from LPS-stimulated injury via inhibiting both apoptosis and necrosis (Figure 6A and B).

\section{Fusu agent treatment suppressed the expression of LPS-induced HPAI in vitro and exerted therapeutic effects in HUVECs by suppressing LPS-induced HPAI}

Then, we assessed the relevance of endothelial HPA1 expression levels in LPS-stimulated HUVECs. We detected change in HPA1 expression under LPS stimulation. As illustrated in Figure 7A, for confirming the effects of Fusu agent on LPS-induced HPA1, HUVECs pretreated with LPS were co-incubated with different doses of Fusu agent-medicated serum for 24 hours. As expected, Fusu agent-medicated serum downregulated HPA1 induced by LPS stimulation in HUVECs (Figure 7A). For figuring out whether upregulated HPA1 by LPS stimulation contributes to cellular injury after LPS stimulation, we knocked down HPA1 induced by LPS and detected cellular injury by measuring LDH leakage. As shown in Figure 7B, without disturbing the ROS accumulation and MTP (data not shown), knockdown of HPA1 partially decreased LDH leakage after LPS stimulation, indicating that LPS-induced HPA1 partially contributes to LPS-induced cellular injury, which is consistent with previous finding. ${ }^{23}$ 
A
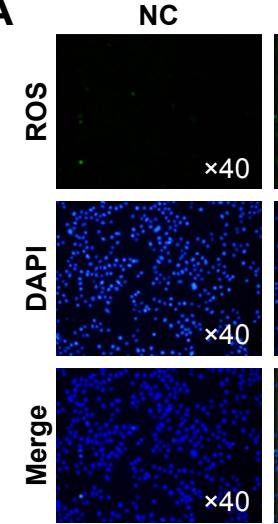

C
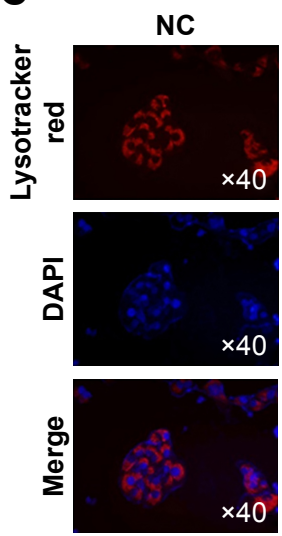

M
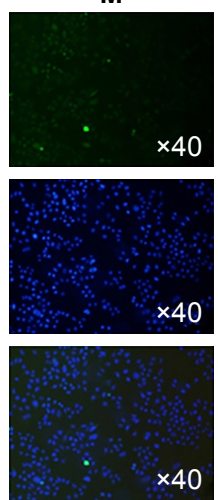

$\times 40$
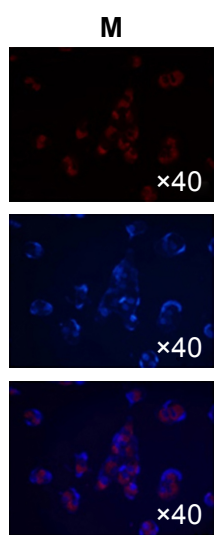

LD
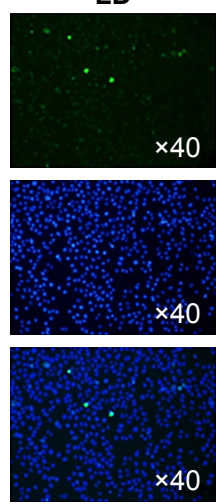

$\times 40$
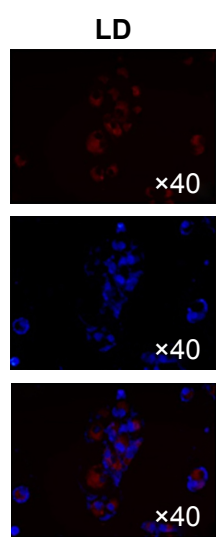

MD
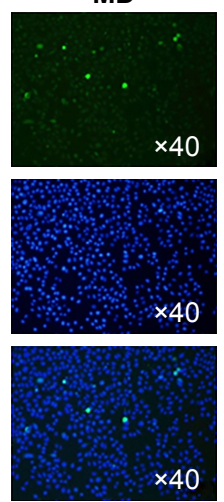

$\times 40$
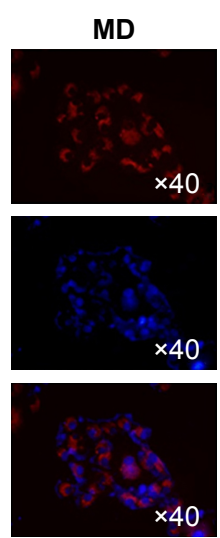

HD
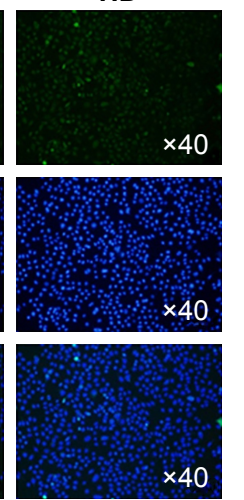

$\times 40$
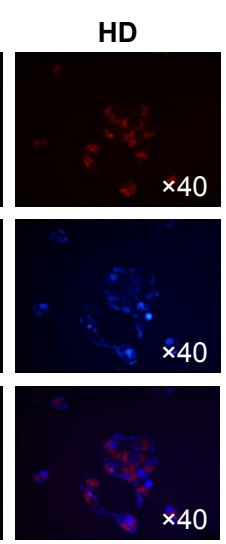

B

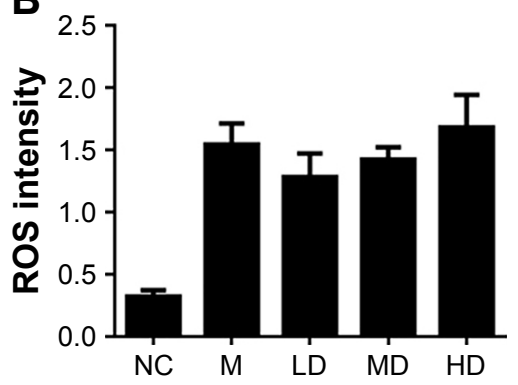

D

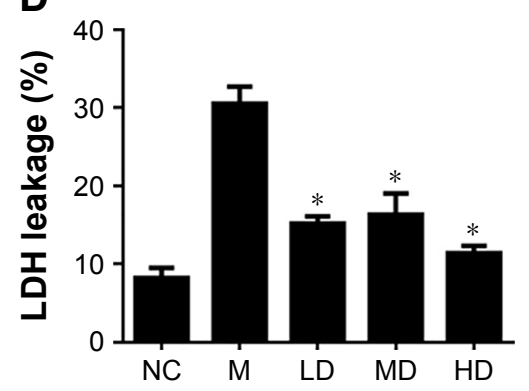

E

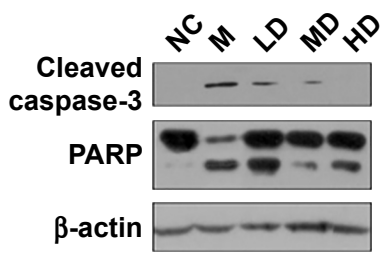

Figure 5 Fusu agent-medicated serum inhibited LPS-induced cellular injury and apoptotic death without affecting ROS accumulation induced by LPS exposure.

Notes: ROS accumulation in NC, M, LD, MD and HD groups was imaged (A) and quantitatively measured (B). (C) Lysotracker staining was performed to detect the MTP affected by Fusu agent-medicated serum. The effects of Fusu agent-medicated serum on LDH leakage (D) and caspase-3 cleavage (E) were detected to demonstrate cell injury. $* P<0.05$ vs M group.

Abbreviations: LPS, lipopolysachharide; MTP, mitochondrial transmembrane potential; LDH, lactate dehydrogenase; NC, negative control group; M, model group; LD, low dose; MD, middle dose; HD, high dose.

It is observed that treatment with Fusu agent downregulated HPA1 expression and protected HUVECs from LPS-induced apoptotic death (Figure 6), and these results promoted us to figure out whether Fusu agent exerts its therapeutic effects via suppressing HPA1. LPS-pretreated HUVECs were incubated with high dose of Fusu agentmedicated serum at a final concentration of $10 \%$ for the next 24 hours. As shown in Figure 7C, consistent with the previous result, both high dose of Fusu agent-medicated serum and siHPA1 decreased LPS-induced HPA1 protein level in HUVECs. Meanwhile, we examined the activation of caspase-3 and PARP using Western blot. The increased levels of activation of caspase- 3 and PARP by LPS induction, compared to untreated group, were suppressed by both Fusu agent-medicated serum exposure and siHPA1 transfection (Figure 7D). On performing Annexin V/PI staining, it was found that LPS exposure induced apoptotic cell death. Both Fusu agent-medicated serum exposure and siHPA1 introduction significantly decreased apoptotic cell death, indicating that Fusu agent-medicated serum protects HUVECs from
LPS-induced apoptotic cell death via downregulating HPA1 (Figure 7E).

Taken together, in LPS-induced ALI model rats, Fusu agent exerts its therapeutic effects and, thus, improves the survival rate. Further research involving HUVECs revealed similar therapeutic effects of Fusu agent-medicated serum, and found that the downregulation of HPA1 by Fusu agent is potentially one of the therapeutic mechanisms both in vivo and in vitro.

\section{Discussion}

ALI is primary characterized by an increase in vascular permeability and pulmonary edema. The degradation of endothelial glycocalyx, which serves as the interface between the vessel wall and the circulating blood, ${ }^{13,32}$ remarkably contributes to the pathophysiology of critical diseases. ${ }^{33-35}$ In ALI induced by inflammation, such as LPS-induced ALI, the direct association of glycocalyx degradation with tissue injury was demonstrated by the increasing plasma concentration of glycocalyx fragments. ${ }^{36,37}$ In the present study, 
A

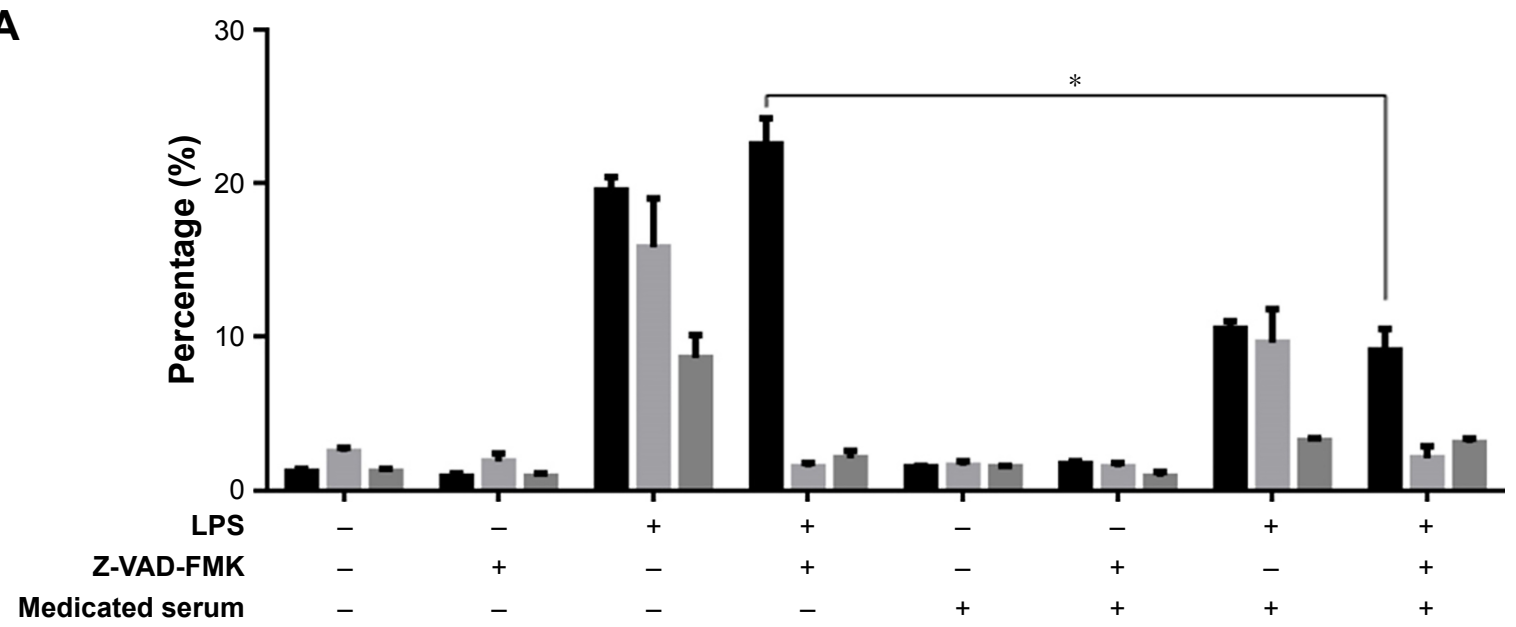

B

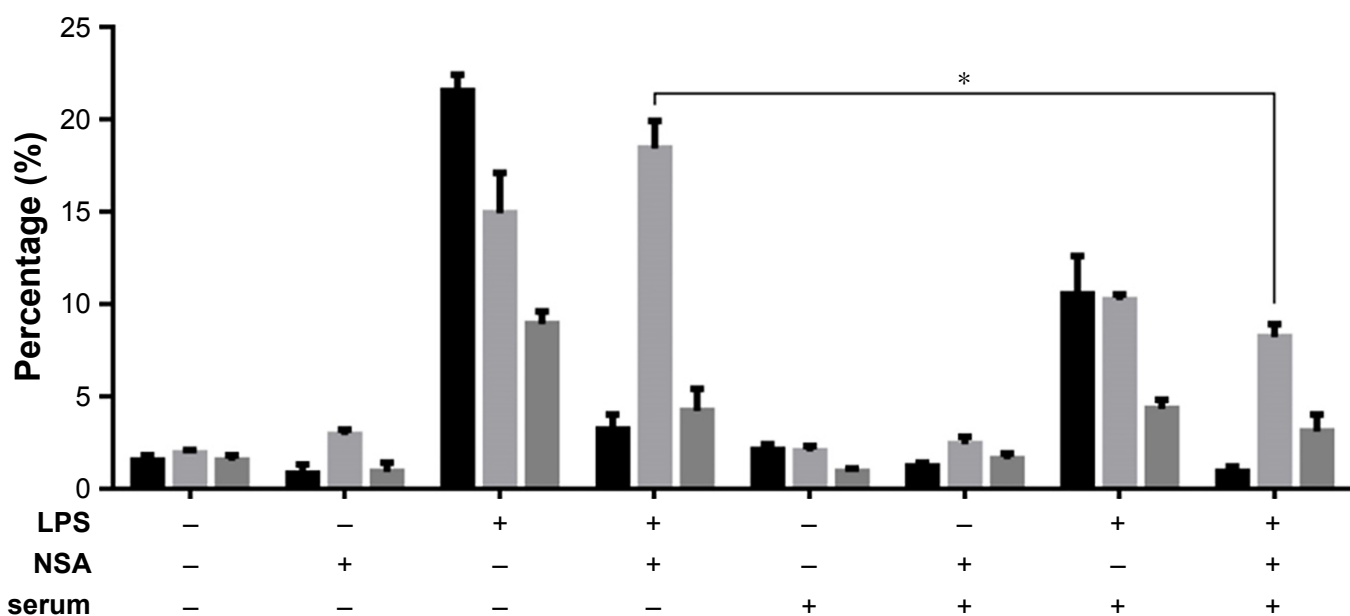

Medicated serum

$-$

Necrosis

Apoptosis

Necrosis+apoptosis

Figure 6 Fusu agent-medicated serum inhibited LPS-induced apoptotic and necrotic cell death in HUVECs.

Notes: Fusu agent-medicated serum protected HUVECs from LPS-induced necrosis (A) and apoptosis (B). $* P<0.05$.

Abbreviations: HUVECs, human umbilical vein endothelial cells; LPS, lipopolysaccharide.

we characterized the in vivo therapeutic effects of the Chinese Herbal Medicine Fusu agent on LPS-induced ALI. We found that Fusu agent could attenuate the pathological changes induced by LPS injection in rat lungs, and the therapeutic effects were dependent on the dose of Fusu agent. We also demonstrated that LPS injection damaged vascular endothelial cells, which is consistent with a previous report, ${ }^{38}$ and that treatment with Fusu agent recovered the vascular endothelial cells from LPS-caused injury. Subsequently, Fusu agenttreated rats presented a remarkably higher survival rate in rat model of LPS-induced ALI.

HPA1 is an endo- $\beta$-glucuronidase that breaks down $\mathrm{HS}$ at specific interchain sites, causing degradation of glycocalyx. ${ }^{31}$ Under normal physiological conditions, HPA1 is expressed in platelets, cytotrophoblasts, mast cells, neutrophils, macrophages, and the placenta. ${ }^{31,39,40}$ It is reported that, HPA1 released by endothelial cells plays a critical role in glycocalyx degradation and, thus, causes ALI after LPS injection. ${ }^{11}$ This promoted us to determine whether Fusu agent exerts its therapeutic effects by regulating the expression of HPA1 in LPS-induced ALI model rats. For this purpose, we found the transcriptional and translational levels of HPA1 after treatment with Fusu agent in LPS-induced ALI model rats. We found that Fusu agent downregulated both HPA1 mRNA and protein levels in LPS-induced rat model, indicating the potential mechanism of its therapeutic effects. However, we failed to detect the effect of Fusu agent on the release of HPA1 after LPS injection (data not shown), potentially due to the low level of HPA1 in alveolar fluid. For further demonstrating the regulation of HPA1 in vascular endothelial cells, HUVECs were employed and were incubated with Fusu agent-medicated serum after LPS exposure. Consistent with the in vivo results, Fusu agent-medicated serum also downregulated HPA1 mRNA and protein levels, which 
A

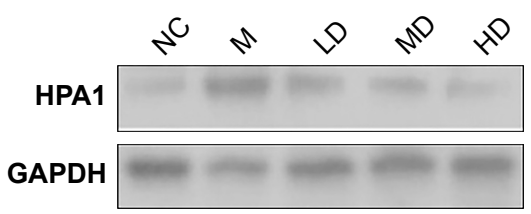

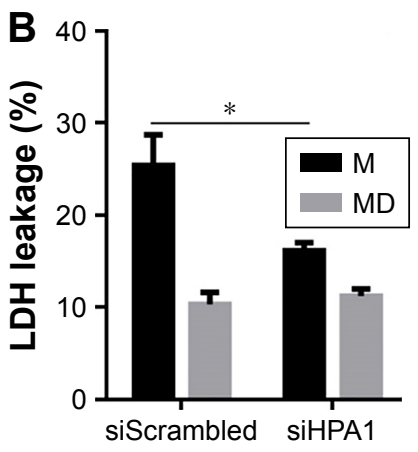

D

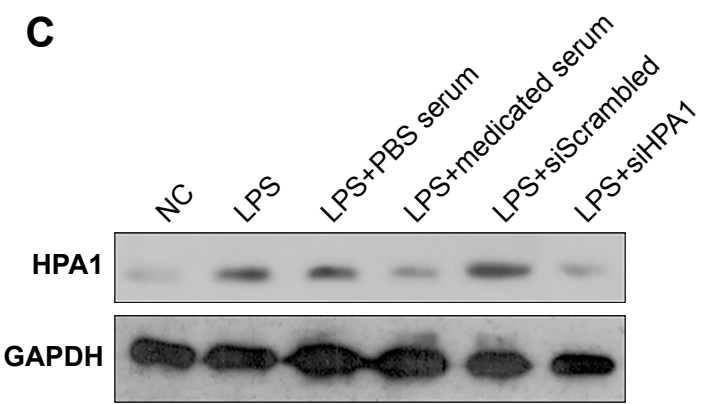

C

NC

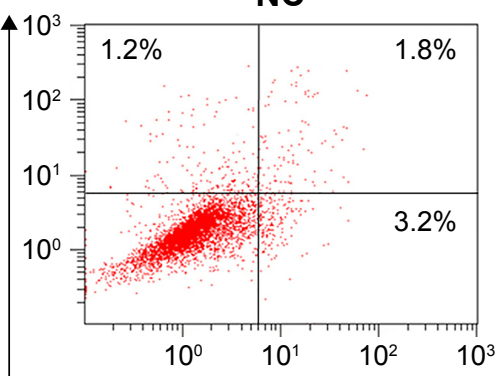

$\bar{\alpha}$

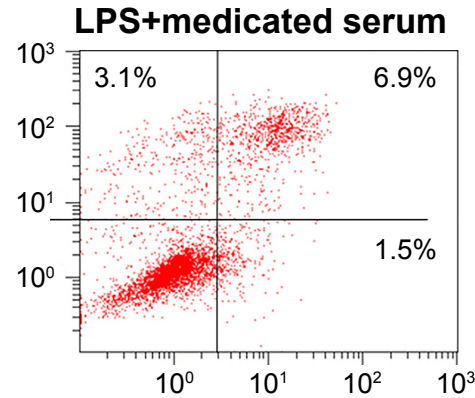

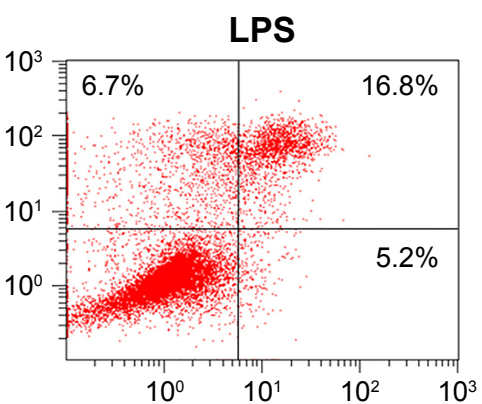

a Uncleaved

$\frac{0}{\underline{\alpha}}$

Cleaved

GAPDH
E

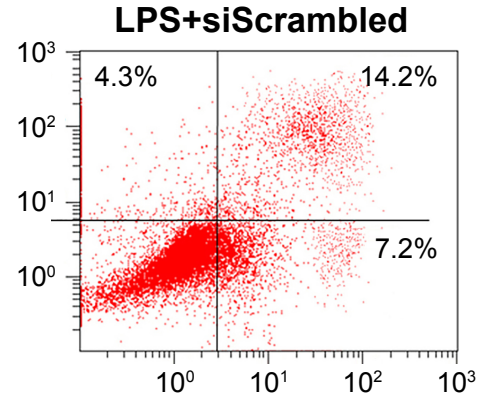

$\begin{array}{llll}10^{0} & 10^{1} & 10^{2} & 10^{3}\end{array}$
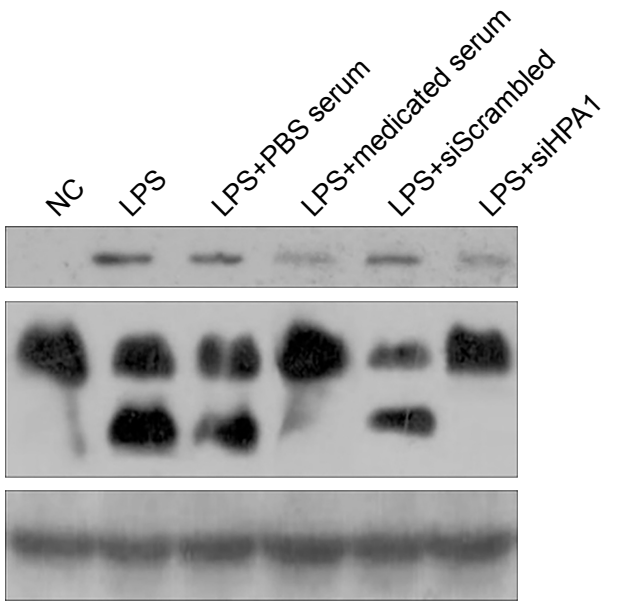
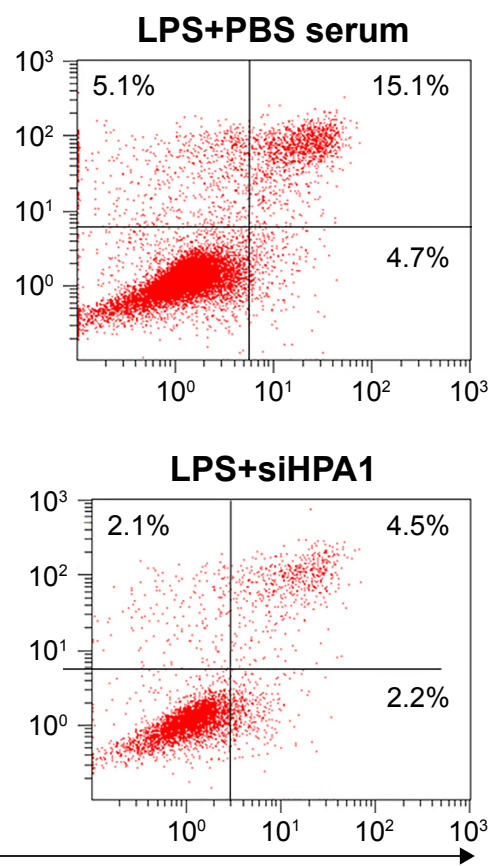

Annexin V-FITC

Figure 7 Fusu agent-medicated serum exerted therapeutic effects mainly via targeting HPAI, which is critical for LPS-induced cell injury.

Notes: (A) After LPS induction, the HPAI protein levels were detected by Western blot in HUVECs. (B) After HPAI knockdown, the therapeutic effects of Fusu agentmedicated serum on LPS-induced HUVECs were determined by measuring LDH leakage. (C) Fusu agent-medicated serum treatment and siHPAI transfection decreased HPAI expression induced by LPS exposure. Both HPAI knockdown and Fusu agent-medicated serum treatment inhibited LPS-induced caspase-3, PARP activity (D) and apoptosis (E). $* P<0.05$ vs SiScrambled group.

Abbreviations: FITC, fluorescein isothiocyanate; HUVECs, human umbilical vein endothelial cells; LPS, lipopolysaccharide; PI, propidium iodide; LDH, Lactate dehydrogenase; HPAI, heparanase I; NC, negative control group. 
further confirmed the potential mechanism of Fusu agent's therapeutic effects.

LPS stimulation is well known to be a critical trigger for endothelial cell activation both in vivo and in vitro. ${ }^{42}$ In vitro, it was reported that LPS stimulation induced functional alterations in the expression and secretion of IL-6 and IL-8 in endothelial cells. ${ }^{41,42}$ In cultured HUVECs, LPS stimulation promoted the inflammation process and caused ROS and NO accumulation, and conversely, pretreatment of ROS/NO scavenger remarkably protected HUVECs from inflammation caused cell injury. ${ }^{13}$ It has been reported that the accumulated ROS caused irreversible damage to ALI rats via disabling mitochondria membrane potential, which is consistent with a previous report showing that excessive ROS can cause mitochondrial dysfunction and leads to caspase-3-dependent apoptotic cell death. ${ }^{11,15,16}$ These data indicate the potential mechanism of LPS-induced lung injury. In this study, we also detected the effects of Fusu agent-medicated serum on this potential mechanism. As expected, LPS stimulation significantly promoted ROS generation, caused instability of mitochondrial membrane potential and activation of capsase- 3 and subsequently caused apoptotic cell death. Treatment of Fusu agent-medicated serum attenuated apoptotic cell death and promoted cell survival rate in HUVECs, indicating its therapeutic effects against LPS stimulation. However, the ROS accumulated by LPS stimulation showed no detectable changes after Fusu agent-medicated serum treatment, indicating that Fusu agent-medicated serum stabilized mitochondrial membrane potential via regulating mitochondrial respiratory chain.

In summary, the present study serves to clarify the role of treatment of Fusu agent or Fusu agent-medicated serum in vivo or in vitro in exerting therapeutic effects in LPSinduced ALI model rats. By performing in vitro experiments on HUVECs, we confirmed the therapeutic effects of Fusu agent-medicated serum on LPS stimulation and revealed the possible mechanism, which is that it stabilizes the mitochondrial membrane potential and apoptotic cell death induced by activated caspase-3 without disturbing the accumulation of ROS. Also, treatment of Fusu agent effectively inhibited the expression of HPA1 in vitro and in vivo, which contributed to endothelial cell damage critically.

\section{Conclusion}

The study proved that Fusu agent exerted its therapeutic effects in LPS-induced lung injury both in vitro and in vivo.

\section{Acknowledgment}

This work was supported by National Natural Science Foundation of China (no 81373836).

\section{Author contributions}

$\mathrm{BL}, \mathrm{ZZ}$ and $\mathrm{CZ}$ contributed to the conception, design and acquisition of data. $\mathrm{CW}, \mathrm{KL}$ and $\mathrm{LG}$ contributed to acquisition, analysis and interpretation of data. PG contributed to the conception and design and gave final approval of the version to be published. All authors contributed to data analysis, drafting and revising the article, gave final approval of the version to be published, and agree to be accountable for all aspects of the work.

\section{Disclosure}

The authors report no conflicts of interest in this work.

\section{References}

1. Ware LB, Matthay MA. The acute respiratory distress syndrome. N Engl J Med. 2000;342(18):1334-1349.

2. Malhotra A. Low-tidal-volume ventilation in the acute respiratory distress syndrome. N Engl J Med. 2007;357(11):1113-1120.

3. Matthay MA, Ware LB, Zimmerman GA. The acute respiratory distress syndrome. J Clin Invest. 2012;122(8):2731-2740.

4. Wheeler AP, Bernard GR. Acute lung injury and the acute respiratory distress syndrome: a clinical review. Lancet. 2007;369(9572): 1553-1564.

5. Rubenfeld GD, Caldwell E, Peabody E, et al. Incidence and outcomes of acute lung injury. $N$ Engl J Med. 2005;353(16):1685-1693.

6. Blank R, Napolitano LM. Epidemiology of ARDS and ALI. Crit Care Clin. 2011;27(3):439-458.

7. Angus DC, Linde-Zwirble WT, Lidicker J, et al. Epidemiology of severe sepsis in the United States: analysis of incidence, outcome, and associated costs of care. Crit Care Med. 2001;29(7):1303-1310.

8. Sheu CC, Gong MN, Zhai R, et al. Clinical characteristics and outcomes of sepsis-related vs non-sepsis-related ARDS. Chest. 2010;138(3): $559-567$.

9. Kong G, Huang X, Wang L, et al. Astilbin alleviates LPS-induced ARDS by suppressing MAPK signaling pathway and protecting pulmonary endothelial glycocalyx. Int Immunopharmacol. 2016;36:51-58.

10. Schmidt EP, Yang Y, Janssen WJ, et al. The pulmonary endothelial glycocalyx regulates neutrophil adhesion and lung injury during experimental sepsis. Nat Med. 2012;18(8):1217-1223.

11. Zhao J, Jiang P, Zhang W. Molecular networks for the study of TCM pharmacology. Brief Bioinform. 2010;11(4):417-430.

12. Vlodavsky I, Iozzo RV, Sanderson RD. Heparanase: multiple functions in inflammation, diabetes and atherosclerosis. Matrix Biol. 2013;32(5):220-222.

13. Liu J, Li G, Chen C, Chen D, Zhou Q. MiR-6835 promoted LPSinduced inflammation of HUVECs associated with the interaction between TLR-4 and AdipoR1 in lipid rafts. PLoS One. 2017;12(11): e0188604

14. Liu XY, Xu HX, Li JK, et al. Neferine protects endothelial glycocalyx via mitochondrial ROS in lipopolysaccharide-induced acute respiratory distress syndrome. Front Physiol. 2018;9:102-115.

15. Li P, Nijhawan D, Budihardjo I, et al. Cytochrome c and dATPdependent formation of Apaf-1/caspase-9 complex initiates an apoptotic protease cascade. Cell. 1997;91(4):479-489.

16. du Q, Wang $C$, Zhang $\mathrm{N}$, et al. In vivo study of the effects of exogenous hydrogen sulfide on lung mitochondria in acute lung injury in rats. $B M C$ Anesthesiol. 2014;14:117-127. 
17. Schon EA, Manfredi G. Neuronal degeneration and mitochondrial dysfunction. J Clin Invest. 2003;111(3):303-312.

18. Reitsma S, Slaaf DW, Vink H, van Zandvoort MA, Oude Egbrink MG. The endothelial glycocalyx: composition, functions, and visualization. Pflugers Arch. 2007;454(3):345-359.

19. Wang Y, Zhang Y, Jiang R. Early traditional Chinese medicine bundle therapy for the prevention of sepsis acute gastrointestinal injury in elderly patients with severe sepsis. Sci Rep. 2017;7:46015.

20. Jiang G, Zheng L, Pu J, et al. Small RNAs targeting transcription start site induce heparanase silencing through interference with transcription initiation in human cancer cells. PLoS One. 2012;7(2):e31379.

21. Wang CX, Gao PY, Xie Q. Effect of modified qianyang pellet on extravascular lung water and its correlated factors at the early fluid resuscitation stage of septic shock. Zhongguo Zhong Xi Yi Jie He Za Zhi. 2011;31(2):200-203.

22. Gao P, Xie Q. The influence of lung capillary leak in eptic shock rat intervene using isotopic tracer method by Fusu preparation. In: China Society of Integrated Traditional Chinese and Western Medicine.

23. Gao P, He C, Zhang C, et al. Preventive effect on endothelial surface layer damage of Fusu agent in LPS-induced acute lung injury in rats. Mol Cell Biochem. 2018;444:1-11.

24. Wei W, Wu XM, Li YJ. Experimental Methodology of Pharmacology. Beijing: People's Medical Publishing House; 2010:389-393.

25. Piali L, Hammel P, Uherek C, et al. CD31/PECAM-1 is a ligand for alpha $\mathrm{v}$ beta 3 integrin involved in adhesion of leukocytes to endothelium. J Cell Biol. 1995;130(2):451-460.

26. Schoenbein C, Docke WD, Wolk K, et al. Long-term interleukin-10 presence induces the development of a novel, monocyte-derived cell type. Clin Exp Immunol. 2008;151(2):306-316.

27. Bao W, Luo Y, Wang D, et al. Sodium salicylate modulates inflammatory responses through AMP-activated protein kinase activation in LPS-stimulated THP-1 cells. J Cell Biochem. 2018;119(1):850-860.

28. Lee HH, Chang ZF. Regulation of RhoA-dependent ROCKII activation by Shp2. J Cell Biol. 2008;181(6):999-1012.

29. Weinbaum S, Tarbell JM, Damiano ER. The structure and function of the endothelial glycocalyx layer. Annu Rev Biomed Eng. 2007;9: 121-167.

30. Han J, Mandal AK, Hiebert LM. Endothelial cell injury by high glucose and heparanase is prevented by insulin, heparin and basic fibroblast growth factor. Cardiovasc Diabetol. 2005;4(4):12-23.
31. Sun B, Zou X, Chen Y, Zhang P, Shi G. Preconditioning of carbon monoxide releasing molecule-derived $\mathrm{CO}$ attenuates LPS-induced activation of HUVEC. Int J Biol Sci. 2008;4(5):270-278.

32. Cohen E, Atzmon R, Vlodavsky I, Ilan N. Heparanase processing by lysosomal/endosomal protein preparation. FEBS Lett. 2005;579(11): 2334-2338.

33. Burke-Gaffney A, Evans TW. Lest we forget the endothelial glycocalyx in sepsis. Crit Care. 2012;16(2):121-122.

34. Johansson PI, Stensballe J, Rasmussen LS, Ostrowski SR. A high admission syndecan-1 level, a marker of endothelial glycocalyx degradation, is associated with inflammation, protein $\mathrm{C}$ depletion, fibrinolysis, and increased mortality in trauma patients. Ann Surg. 2011;254(2):194-200.

35. Myburgh JA, Mythen MG. Resuscitation fluids. N Engl J Med. 2013; 369(13):1243-1251.

36. Nelson A, Berkestedt I, Schmidtchen A, Ljunggren L, Bodelsson M. Increased levels of glycosaminoglycans during septic shock: relation to mortality and the antibacterial actions of plasma. Shock. 2008; 30(6):623-627.

37. Steppan J, Hofer S, Funke B, et al. Sepsis and major abdominal surgery lead to flaking of the endothelial glycocalix. J Surg Res. 2011;165(1): $136-141$.

38. Keshari RS, Silasi-Mansat R, Zhu H, et al. Acute lung injury and fibrosis in a baboon model of Escherichia coli sepsis. Am J Respir Cell Mol Biol. 2014;50(2):439-450.

39. Freeman C, Browne AM, Parish CR. Evidence that platelet and tumour heparanases are similar enzymes. Biochem J. 1999;342 (Pt 2): 361-368.

40. Jehle AB, Li Y, Stechschulte AC, et al. Endotoxin and mast cell granule proteases synergistically activate human coronary artery endothelial cells to generate interleukin-6 and interleukin-8. J Interferon Cytokine Res. 2000;20(4):291-297.

41. von Asmuth EJ, Leeuwenberg JF, Ceska M, Buurman WA. LPS and cytokine-induced endothelial cell IL-6 release and ELAM-1 expression: involvement of serum. Eur Cytokine Netw. 1991;2(4):291-297.

42. Charreau B, Coupel S, Goret F, Pourcel C, Soulillou JP. Association of glucocorticoids and cyclosporin A or rapamycin prevents E-selectin and IL-8 expression during LPS- and TNFalpha-mediated endothelial cell activation. Transplantation. 2000;69(5):945-953.
Drug Design, Development and Therapy

\section{Publish your work in this journal}

Drug Design, Development and Therapy is an international, peerreviewed open-access journal that spans the spectrum of drug design and development through to clinical applications. Clinical outcomes, patient safety, and programs for the development and effective, safe, and sustained use of medicines are the features of the journal, which

\section{Dovepress}

has also been accepted for indexing on PubMed Central. The manuscript management system is completely online and includes a very quick and fair peer-review system, which is all easy to use. Visit http://www.dovepress.com/testimonials.php to read real quotes from published authors. 